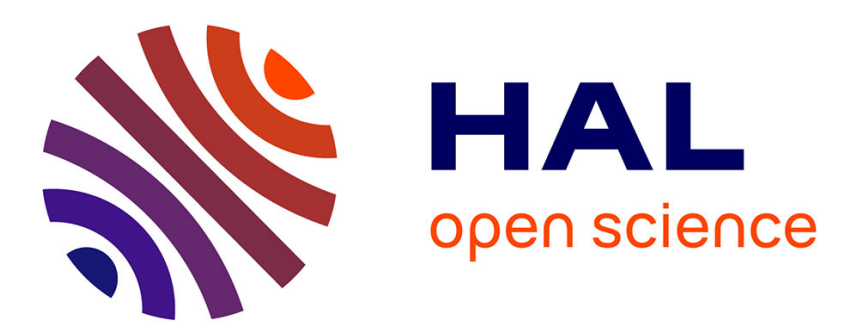

\title{
A Connected-Tube MPP Model for Object Detection with Application to Materials and Remotely-Sensed Images
}

Tianyu Li, Mary Comer, Josiane Zerubia

\section{- To cite this version:}

Tianyu Li, Mary Comer, Josiane Zerubia. A Connected-Tube MPP Model for Object Detection with Application to Materials and Remotely-Sensed Images. ICIP 2018 - IEEE International Conference on Image Processing, Oct 2018, Athens, Greece. hal-02157080

\section{HAL Id: hal-02157080 \\ https://hal.inria.fr/hal-02157080}

Submitted on 18 Jun 2019

HAL is a multi-disciplinary open access archive for the deposit and dissemination of scientific research documents, whether they are published or not. The documents may come from teaching and research institutions in France or abroad, or from public or private research centers.
L'archive ouverte pluridisciplinaire HAL, est destinée au dépôt et à la diffusion de documents scientifiques de niveau recherche, publiés ou non, émanant des établissements d'enseignement et de recherche français ou étrangers, des laboratoires publics ou privés. 


\section{A CONNECTED-TUBE MPP MODEL FOR OBJECT DETECTION WITH APPLICATION TO MATERIALS AND REMOTELY-SENSED IMAGES}

\author{
Tianyu Li, Mary Comer \\ School of Electrical and Computer Engineering \\ Purdue University \\ West Lafayette, IN, USA
}

\author{
Josiane Zerubia \\ Université Côte d'Azur \\ Inria \\ Sophia Antipolis Cedex, France
}

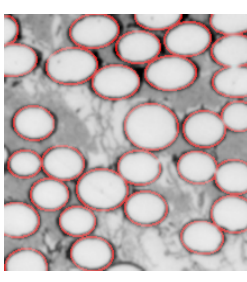

(a)

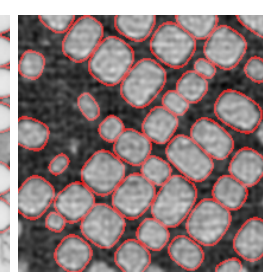

(b)

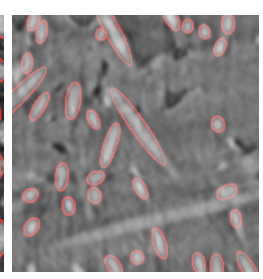

(c) MPP model for more complex detection tasks. The proposed tube model is applied to fiber detection in microscopy images by combining connected-tube and ellipse models. The ellipse model is used for detecting short fibers, while the longer fibers are detected by tube model. We also test the model on road and building detection in remotely sensed images.

Index Terms - Mixed Marked Point Process, stochastic modeling, connected tube model, fiber detection, road and building detection

\section{Introduction}

For the characterization of microscope images of materials, stochastic image models, such as the Markov Random Field [1,2] and the Marked Point Process (MPP) [3], have been shown to be very promising. This paper proposes a new MPP shape model for the analysis of fiber-reinforced composite materials $[4,5]$. Fibers are modeled as geometric objects forming a point process [6-8] with marks for each object. The target object configuration can be sampled by the Markov Chain Monte Carlo (MCMC) based birth and death sampler [9].

Previously proposed MPP shape models work well when the objects have similar size and shape in images, such as those shown in Figure1(a) and Figure1(b) which use ellipses and super ellipses. However, these may not work well with fibers whose size vary over a wide range, as shown in Figure1(c), which miss the longest fiber. The approach is therefore both time-consuming and inaccurate in detection of fibers. There are mainly two reasons for the poor performance of MPP in this application. First, the long fiber can not be described by ellipse accurately. Second, the large variation
Fig. 1: (a) MPP result on ellipse fiber detection ; (b) MPP result on super ellipse fiber detection; (c) MPP result on long fiber detection.

of the fiber's size increases the searching time.

In order to solve the problem of detecting long fibers in Figure1(c), we propose a connected-tube model based on a Marked Point Process. In our approach, instead of modeling the long fiber by an ellipse object, we model it as a series of connected tubes. Since the long fibers are modeled by connected tubes, the size of the tubes can be controlled in a smaller range.

The tube model can be combined easily with the ellipse or rectangle model to form a mixed MPP model. Compared with Lafarge et al. [10]'s approach, we replace the segments and lines by tubes, which have similar parameters and energy term as the ellipses, and the switching between tube and ellipse is simpler.

Moreover, we show that the connected-tube model can also be applied to road detection in 2D remotely sensed images, which has been a popular topic in recent years [11-14]. When combined with a rectangle model, it is possible to detect the buildings and roads at the same time. Compared with the previously proposed Quality Candy model [15], our tube model has a much simpler prior for connection.

The paper is organized as follows: In Section 2, we describe the proposed tube model under a mixed MPP framework. In Section 3, the optimization method is presented. In Section 4, experimental results for fiber detection in microscopic image, road and building detection in remotely sensed images are given. Conclusions are outlined in Section 5. 


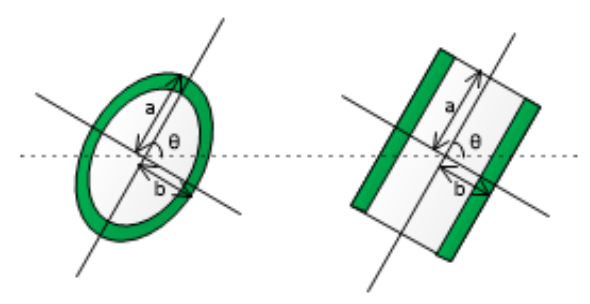

Fig. 2: Ellipse and Tube models

\section{A Mixed Marked Point Process of Ellipses and Tubes}

The framework of the Mixed Marked Point Process of ellipses and tubes is similar to the framework of general multimarked point process in [10]. The point space is defined on an image lattice $S=\left[0, X_{\max }\right] \times\left[0, Y_{\max }\right], S \subset \mathbb{R}^{2}$. The mark space $M$ is defined as the union of the ellipse mark set $M_{e}$ and the tube mark set $M_{t}$, so $M=M_{e} \cup M_{t}$. In our model, an ellipse object and a tube object have similar random variables for each mark, which makes it easier to switch between them, compared to the switch between an ellipse and a segment in [10]. Their shapes can be seen in Figure 2, where a,b are the major and minor axes which control the size of the ellipse and the tube respectively, and $\theta$ controls the orientation. Here, $M_{e}=M_{t}=\left[a_{\min }, a_{\max }\right] \times\left[b_{\min }, b_{\max }\right] \times[0, \pi]$, for some parameters $a_{\min }, a_{\max }, b_{\min }, b_{\max }$.

A random marked object field $W$ is a subset of $S \times M$. A marked object is defined as a vector $W_{i}=\left(S_{i}, M_{i}\right) \in W$. Let $\Omega_{W}$ be the configuration space, which denotes the space of all possible realizations of $W$. Then $w=\left(w_{1}, \ldots, w_{n}\right) \in \Omega_{W}$ is a possible object configuration, where $n$ is the number of objects in this configuration. Note the number of objects in the random field $W$ is a random variable and could be quite a large number. Let $Y$ be the observed image. Then the density of the mixture marked point process is given by

$$
f(w \mid y)=\frac{1}{Z} \exp \left\{-V_{d}(y \mid w)-V_{p}(w)\right\}
$$

where $Z$ is the normalizing constant, $V_{d}(y \mid w)$ is the data energy, which describes how well the objects fit the observed image, $V_{p}(w)$ is the prior energy introducing the prior knowledge on the object configuration.

\subsection{Data Energy}

Data energy $V_{d}(y \mid w)$ is the sum of the individual object energies:

$$
V_{d}(y \mid w)=\sum_{i} V_{d}\left(y \mid w_{i}\right)
$$

where $V_{d}\left(y \mid w_{i}\right)$ describes how well object $w_{i}$ fit the observed image $y$. We associate an inner region $D_{i n}$ and outer region
$D_{\text {out }}$ with each object, shown as the white area and green area inside objects in Figure 2, respectively. The outer area is set to be 2 pixels wide, good compromise for all the images tested. By defining the Bhattacharya distance $B\left(y \mid w_{i}\right)$ between the inner region and outer region of each object, $V_{d}\left(y \mid w_{i}\right)$ can be calculated as in [16]:

$$
V_{d}\left(y \mid w_{i}\right)= \begin{cases}1-\frac{B\left(y \mid w_{i}\right)}{T} & B\left(y \mid w_{i}\right)<T \\ \exp \left(-\frac{B\left(y \mid w_{i}\right)-T}{3 B\left(y \mid w_{i}\right)}\right)-1 & \text { otherwise }\end{cases}
$$

\subsection{Prior Energy}

$V_{p}(w)$ describes the prior knowledge about objects. It is given as

$$
V_{p}(w)=\alpha V_{p}^{o l}(w)+\beta V_{p}^{l e n}(w)+\gamma V_{p}^{c o n}(w)
$$

where $V_{p}^{o l}(w)$ penalizes overlapping between objects, $V_{p}^{\text {len }}(w)$ penalizes the tubes with short length (the axis a); $V_{p}^{c o n}(w)$ encourages connections between tubes; and $\alpha, \beta$ and $\gamma$ are weights for each term, which are set by trial and error.

\subsubsection{Overlap Prior}

The overlap prior $V_{p}^{o l}(w)$ is applied to all objects.

$$
\begin{gathered}
V_{p}^{o l}(w)=\sum_{i, j} V_{p}^{o l}\left(w_{i}, w_{j}\right) \\
V_{p}^{o l}\left(w_{i}, w_{j}\right)= \begin{cases}R\left(w_{i}, w_{j}\right) & \text { if } R\left(w_{i}, w_{j}\right)<T_{o l} \\
\infty & \text { else }\end{cases}
\end{gathered}
$$

where $R\left(w_{i}, w_{j}\right)$ is the mutual overlap ratio between object $w_{i}$ and $w_{j}$ as in [3]. $T_{o l}$ is the overlap threshold. It is set to 0.25 in our experiments.

\subsubsection{Length Prior}

Shorter tubes may over fit the observed image $Y$, which could unreasonably increase the dimension of object configuration $w$. So we introduce $V_{p}^{l e n}(w)$ to penalize the short tube objects. We let

$$
V_{p}^{l e n}(w)=\sum_{w_{i} \in w_{T}} V_{p}^{l e n}\left(w_{i}\right)
$$

where $w_{T}$ is the collection of tube objects in $w, V_{p}^{\text {len }}\left(w_{i}\right)=$ $\exp \left(\left(a_{\max }-a_{i}\right) / a_{\max }\right)$, and $a_{i}$ is the major axis $a$ of tube object $w_{i}$.

\subsubsection{Connection Prior}

To encourage the tubes to be connected, we introduce the connect prior $V_{p}^{c o n}(w)$.

$$
V_{p}^{c o n}(w)=\sum_{w_{i} \in w_{T}} V_{p}^{c o n}\left(w_{i}\right)
$$




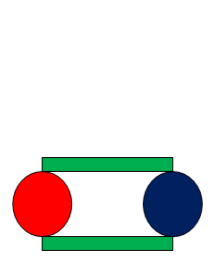

(a)

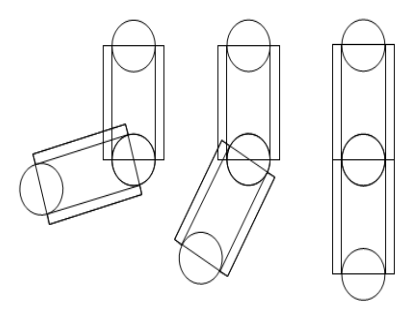

(b)
Fig. 3: (a) Joint area of a tube; (b) Illustration of connection relationship between tubes.

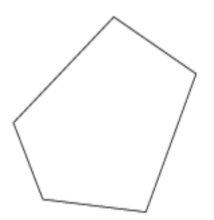

(a)

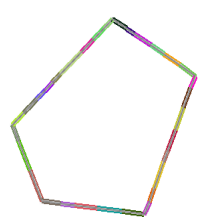

(b)

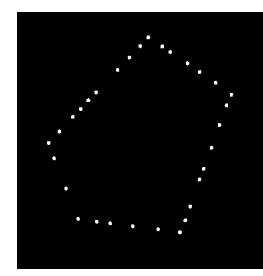

(c)
Fig. 4: (a) Polygon image; (b) Tubes detection result; (c) The connection map.

We define the front and back joint area at the two ends of a tube, as in Figure 3(a), where the blue part is the front joint area and the red part is the back joint area. Unlike the prior model used in the Quality Candy Model [15], we have no free, single or double connection relationship for tubes. Also we do not have orientation potential term for the alignment of tubes. From Figure 3(b), if the angle of two connected tubes is large, the overlapped area would be also large, which will penalize the orientation difference between two connected tubes.

In order to calculate the connection prior, we adopt a connection map, which has the same size as the observed image, to record the joint area of tubes in an object configuration. The map is initialized with zeros. When a tube is generated, the positions that correspond to the joint area of the tubes are incremented by one. Figure 4 shows an example of a connection map.

With the connection map, the connection prior for each tube object $V_{p}^{c o n}\left(w_{i}\right)$ can be calculated as

$$
V_{p}^{c o n}\left(w_{i}\right)=0.5 \times F^{c o n}\left(w_{i}\right)+0.5 \times B^{c o n}\left(w_{i}\right)
$$

where

$$
\begin{aligned}
& F^{c o n}\left(w_{i}\right)= \begin{cases}1-\frac{R_{f}\left(w_{i}\right)}{T_{c o n}} & \text { if } R_{f}\left(w_{i}\right)<T_{c o n} \\
-\frac{\sqrt{R_{f}\left(w_{i}\right)}-\sqrt{T_{c o n}}}{1-\sqrt{T_{c o n}}} & \text { else }\end{cases} \\
& B^{\text {con }}\left(w_{i}\right)= \begin{cases}1-\frac{R_{b}\left(w_{i}\right)}{T_{\text {con }}} & \text { if } R_{b}\left(w_{i}\right)<T_{c o n} \\
-\frac{\sqrt{R_{b}\left(w_{i}\right)}-\sqrt{T_{c o n}}}{1-\sqrt{T_{c o n}}} & \text { else }\end{cases}
\end{aligned}
$$

$R_{f}\left(w_{i}\right)$ is the connecting overlap ratio of a tube's front joint region in the connection map. $R_{b}\left(w_{i}\right)$ is the connecting overlap ratio of the tube's back joint region on connection map. $T_{\text {con }}$ is the connection threshold, which is set to 0.15 in our experiments. These equations are used for penalizing connections under $T_{c o n}$, and encouraging a high connection rate.

\section{Optimization Method}

The conventional RJ MCMC algorithm [17] is used for finding the optimal configuration that minimizes the energy function $V(w \mid y)=V_{d}(w \mid y)+V_{p}(w)$. State transitions in the configuration space are realized by three types of kernel: Birth and death kernel, perturbation kernel and switch kernel.

Birth and death kernel This kernel allows a dimension change in a configuration by adding or removing an object from $w$ [9]. There are two types of objects in our configuration space. Tubes and ellipses are given the same probability for birth.

Perturbation kernel This kernel changes the marks $a_{i}, b_{i}, \theta_{i}$ for each object $w_{i}$ in $w$. We choose Gaussion distributions for updating the marks [17].

Switch kernel This kernel allows transition between tube objects and ellipse objects. Since we use the same marks for tubes and ellipses, the switch is simple. From tube object $w_{t}=\left(x_{t}, y_{t}, a_{t}, b_{t}, \theta_{t}\right)$ to an ellipse object $w_{e}=$ $\left(x_{e}, y_{e}, a_{e}, b_{e}, \theta_{e}\right)$, we simply let $x_{e}=x_{t}, y_{e}=y_{t}, b_{e}=b_{t}$, $\theta_{e}=\theta_{t}, a_{e}=a_{t}+X$, where $X \sim N(0,4)$. A switch from an ellipse object $w_{e}$ to a tube object $w_{t}$ is made in the same way. In order to speed up the convergence, it is possible to use a Multiple Birth and Death algorithm [16, 18] instead of the classical RJMCMC [17]. This is what we did in the experiments.

\section{Experiments}

We apply the mixed MPP model to a series of fiber-reinforced composite materials images provided by industry. A comparison between our model and an ellipse-only MPP model is given. Also we test on some cut remotely sensed images from dataset [19] to show its potential to detect road network and buildings.

\subsection{Fiber Detection}

In the fiber detection tests, the parameters of our model are set as $T=50, T_{o l}=0.25, T_{\text {con }}=0.15, a_{\min }=4, a_{\max }=22$, $b_{\min }=2, b_{\max }=8, \alpha=0.5, \beta=0.12, \gamma=0.38$. These parameters were determined experimentally to provide good 


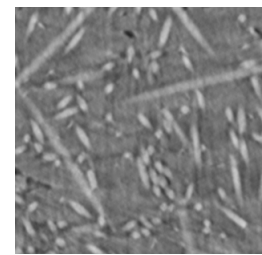

(a)

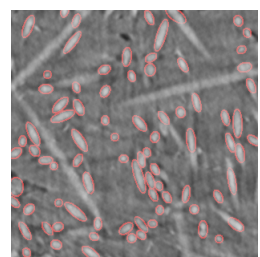

(c)

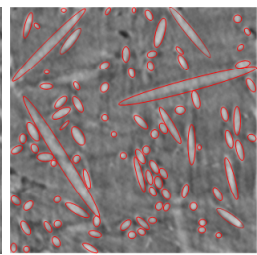

(b)

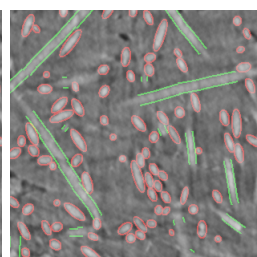

(d)

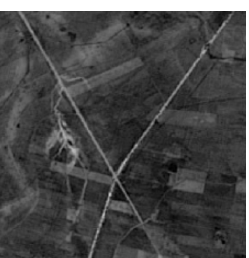

(a)

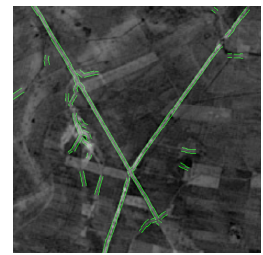

(d)

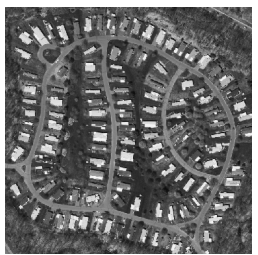

(b)

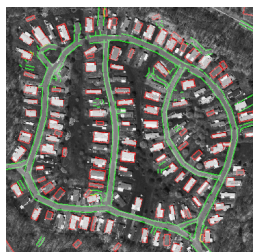

(e)

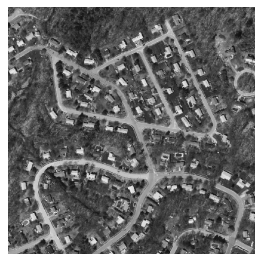

(c)

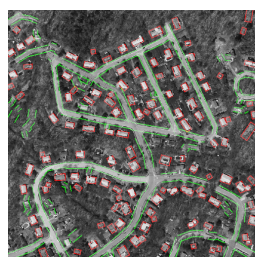

(f)
Fig. 5: (a)Fiber-Reinforced Composite Materials image (Courtesy of Prof. Mike Sangid, Purdue University); (b) Hand drawn fibers (c) Ellipse-only MPP model result; (d) Mixed MPP model result.

results and are kept constant for all material images. Figure 3 presents the results of fiber detection in a microscopy image with dimension $308 \times 308$. From Figure 5(a), the ellipse-only MPP model, with long axis ranging from 2 to 70 pixels misses almost all long fibers, while our model can extract the long fibers. We use 10 images with dimension $308 \times 308$ to verify the performance of our model. The missed long fiber (with $a_{\max } \geq 18$ ) rate, missed short fiber (with $a_{\max }<18$ ) rate, and over detection rate are listed Table 1 . Our model not only outperforms the ellipse-only model for the long fiber detection but also has lower missed detection rate. We believe this is because some short fibers can not be modeled as ellipses accurately. Unfortunately, this will also lead to higher false detection rate since fibers are modeled by two kinds of shape.

\begin{tabular}{|l|l|l|l|}
\hline & $\begin{array}{l}\text { Missed } \\
\text { detection } \\
\text { rate for } \\
\text { long fibers }\end{array}$ & $\begin{array}{l}\text { Missed } \\
\text { detection } \\
\text { rate for } \\
\text { short fibers }\end{array}$ & $\begin{array}{l}\text { False } \\
\text { detection } \\
\text { rate }\end{array}$ \\
\hline ellipse MPP & $81.53 \%$ & $4.22 \%$ & $2.10 \%$ \\
\hline mixed MPP & $9.23 \%$ & $1.40 \%$ & $4.89 \%$ \\
\hline
\end{tabular}

Table 1: Fiber Detection Evaluation

\subsection{Road Detection}

In the road detection problem, we test the proposed model on remotely sensed images to show the potential of it. Figure 6(a), (b), and (c) show three satellite images of road networks, trees, and buildings. Figure $6(\mathrm{~d})$ shows the detection result by connected-tube model. In Figure 6(e), (f), we applied the mixed MPP model by replacing the ellipse model with a rect-
Fig. 6: Road detection. The upper row, original satellite images; the bottom row: our test result.

angle model for detecting buildings.

\section{Conclusion}

For extracting long strip features in images, we have proposed a connected-tube model based on marked point processes. In order to make the tubes connected well, a length prior and a connection prior are introduced. For fiber detection the tube model works well, when combined with the ellipse model in a mixed MPP framework. The tube model aims at detecting long fibers, while the ellipse model can detect short fibers. Since we use the same parameters to control the size and orientation of tubes and ellipses, switching between them is convenient. This mixed model solves the problem of wide mark range in MPP model for detecting the fibers in microscopy image, and improves the accuracy of detecting. We present the result on fiber-reinforced composite materials images to show the advantages of our model. Furthermore the tests of our model on remotely sensed images shows its potential of detecting roads and buildings. More tests will be made in near future.

\section{Acknowledgment}

This research is supported by the Air Force Office of Scientific Research, MURI contract \# FA9550-12-1-0458 and by the National Science Foundation(NSF) grant NSF CMMI MoM 16-62554.

J. Zerubia would like to thank the IEEE Signal Processing Society for financial support as IEEE SPS Distinguished Lecturer in 2016-2017 which gave her the opportunity to meet with Prof. M. Comer and start this collaboration. 


\section{References}

[1] J. P. Simmons, B. Bartha, M. D. Graef, and M. Comer, "Development of bayesian segmentation techniques for automated segmentation of titanium alloy images," $\mathrm{Mi}$ croscopy and Microanalysis, vol. 14(S2), pp. 602-603, 2008.

[2] J. P. Simmons, P. Chuang, M. Comer, J. E. Spowart, M. D. Uchic, and M De Graef, "Application and further development of advanced image processing algorithms for automated analysis of serial section image data," Modelling and Simulation in Materials Science and Engineering, vol. 17, no. 2, pp. 025002, 2009.

[3] H. Zhao, M. L. Comer, and M. De Graef, "A unified markov random field/marked point process image model and its application to computational materials," pp. 6101-6105, 2014.

[4] J. Wang and B. Sharma M. D. Sangid F. Costa X. Jin C. L. Tucker III L. S. Fifield B. N. Nguyen, R. Mathur, "Fiber orientation in injection molded long carbon fiber thermoplastic composites," in Proceedings of the Technical Conference \& Exhibition, 2015.

[5] R. F. Agyei, B. Sharma, and M. D. Sangid, "Investigating sub-surface microstructure in fiber reinforced polymer composites via x-ray tomography characterization," in 57th AIAA/ASCE/AHS/ASC Structures, Structural Dynamics, and Materials Conference, 2016.

[6] A. J. Baddeley and M. N. M. Van Lieshout, "Stochastic geometry models in high-level vision," Journal of Applied Statistics, vol. 20, Issue 5-6, pp. 231-256, 1993.

[7] X. Descombes and J. Zerubia, "Marked point process in image analysis," IEEE Signal Processing Magazine, vol. 19, Issue 5, pp. 77-84, 2002.

[8] J. Illian, A. Penttinen, H. Stoyan, and D. Stoyan, Statistical Analysis and Modelling of Spatial Point Patterns, Wiley-Interscience, 2008.

[9] C. J. Geyer and J. Møller, "Simulation and likehood inference for spatial point processes," Scandinavian Journal of Statistics, vol. 21, no. 4, pp. 359-373, 1994.

[10] F. Lafarge, G. Gimel farb, and X. Descombes, "Geometric feature extraction by a multimarked point process," IEEE Transactions on Pattern Analysis and Machine Intelligence, vol. 32, Issue 9, pp. 1597-1609, 2010.

[11] J. D. Wegner, J. Montoya, and K. Schindler, "Road networks as collections of minimum cost paths," ISPRS Journal of Photogrammetry and Remote Sensing, vol. 108, pp. 128-137, 2015.
[12] S. G. Jeong, Y. Tarabalka, N. Nisse, and J. Zerubia, "Progressive Tree-like Curvilinear Structure Reconstruction with Structured Ranking Learning and Graph Algorithm,"〈hal-01414864〉, 2016.

[13] A. Schmidt, F. Lafarge, C. Brenner, F. Rottensteiner, and C. Heipke, "Forest point processes for the automatic extraction of networks in raster data," ISPRS Journal of Photogrammetry and Remote Sensing, vol. 126, pp. 38-55, 2017.

[14] G. Moser and J. Zerubia (Ed.), Mathematical Models for Remote Sensing Image Processing, Springer International Publishing, 2018.

[15] C. Lacoste, X. Descombes, and J. Zerubia, "Point processes for unsupervised line network extraction in remote sensing," IEEE Transactions on Pattern Analysis and Machine Intelligence, vol. 27, Issue 10, pp. 15681579, 2005.

[16] X. Descombes, R. Minlos, and E. Zhizhina, “Object extraction using a stochastic birth-and-death dynamics in continuum," Journal of Mathematical Imaging and Vision, vol. 33, Issue 3, pp. 347-359, 2009.

[17] P. J. Green, "Reversible jump markov chain monte carlo computation and bayesian model determination," Biometrika, vol. 82, Issue 4, pp. 711-732, 1995.

[18] Xavier Descombes, "Multiple objects detection in biological images using a marked point process framework," Methods, vol. 115, pp. 2-8, 2017.

[19] V. Mnih, Machine Learning for Aerial Image Labeling, Ph.D. thesis, University of Toronto, 2013. 